\title{
Micromechanics and Mesomechanics of Laminates: Synergy and Model Improvements
}

\author{
P. Ladevèze, G. Lubineau, D. Marsal \\ LMT-Cachan (E.N.S. de Cachan / Paris 6 Univ. / C.N.R.S) \\ 61 Avenue du Président Wilson 94235 Cachan Cedex France \\ ladeveze@lmt.ens-cachan.fr, lubineau@lmt.ens-cachan.fr
}

\section{SUMMARY}

A systematic relationship bridging micromechanics and the damage mesomodel for laminates which originated at LMT-Cachan has been developed in recent works. The main degradation scenarios were taken into account, the link being defined under the hypothesis of plane macrostresses. Here, this bridge is extended with the introduction of out-of-plane loading. The method remains the same. First, the reference "micro" geometry is defined, including the different scenarios on the microscale. The next step consists of calculating a good approximation based on the resolution of two basic problems - one associated with the ply, the other with the interface. The result is a mesodescription of the microscale based on quasi-intrinsic quantities.

\section{INTRODUCTION}

Today, the modeling and simulation of the mechanisms of damage in laminated composite structures under complex loading until fracture is still a challenge. Damage refers to the more or less progressive growth of microvoids or microcracks leading to macrocracks and, eventually, to fracture. One approach for studying these degradations is to use what we call a "damage mesomodel for laminates". This mesomodel, in which the microcracks and microdebondings have been homogenized, was identified on numerous materials for aeronautics and aerospace applications. The micromechanics of laminates has also been developed, leading to numerous theoretical and experimental works published in the last twenty years ([1], [2], [3], [4], [11]). However, until now, the micromechanics of laminates was far from complete for the prediction of final fracture and localization. Here, our objective is to build a rather general bridge between the micromechanics and the mesomechanics of laminates. 
The main concept on which this damage mesomodel is based is the use of an intermediary scale related to the scale of the laminate. On this intermediary scale, which we call the "mesoscale", the material is described by means of two basic mesoconstituents: the single layer and the interface (Figure 1). Damage mechanics and qualitative microanalysis are used to describe the degradation of the mesoconstituents. Mesodamage indicators are linked to the variation of stiffness of the mesoconstituents. Aside from fiber breakage, the damage mechanisms taken into consideration are matrix microcracking, fiber/matrix debonding and delamination. The interface is a surface entity which depends on the relative orientations of the adjacent plies. It transfers displacements and normal stresses from one ply to another. Preliminary identifications of each mesoconstituent were performed in previous works ([5] for the single layer; [6] for the interface).

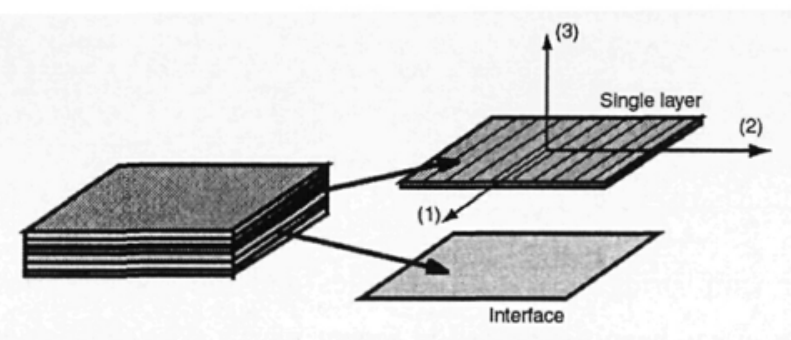

Fig. 1: Decomposition of a laminated composite into mesoconstituents

Another important point is that the state of damage is assumed to be uniform throughout the thickness of each mesoconstituent [7]. The state of damage is described as a constant throughout the thickness of the single layer. Of course, the state of damage within the laminate can vary from one layer to the next. Thus, by using models of these two mesoconstituents, it is possible to reproduce the behavior of any laminate for any loading.

The belief that a complete bridge between the micro- and mesomechanics of laminates exists is not shared by all people working in micromechanics, even though some partial results have been obtained. A first attempt at building such a bridge was made recently for plane macrostresses [8,9]: the mechanisms considered were transverse microcracking (with or without "diffuse" delamination microcracks) originating at the tips of the transverse cracks, and fiber/matrix debonding. The mesomodel was found to be fully compatible with these damage micromechanisms. Micro-meso relations were also derived; these relations introduce quantities or expressions, which we call "approximately ply-material", which are intrinsically related to the cracked ply's characteristics and, therefore, independent of the characteristics of the other plies. The bridge consists of a 2D elasticity problem for each particular stacking sequence. The proof was based on numerous simulations of different possible stacking sequences, thicknesses, etc.

Here, we try to go one step further and introduce the basis of a complete bridge by considering stresses which are not plane. The method of investigation remains the same: numerous numerical experiments (involving possible stacking sequences, thicknesses, etc.) are performed. We show that the microdamage 
within each layer and each interface can be homogenized; relations between the "micro" and "meso" quantities are derived. Such a complete link between the mesomechanics and micromechanics of laminates relies on the resolution of two basic problems: the extension to non-plane stresses of the $2 \mathrm{D}$ problem introduced previously for a particular stacking sequence, plus a new problem which we must introduce to define the homogenized interface. The latter is a 3D problem with periodic conditions, which can be approximated by two 2D problems. Several meso quantities can be easily interpreted in terms of the exact 3D solution: both the plane part of the mesostrain and the out-of-plane part of the stress can be viewed as mean values.

It seems that, again, intrinsic relationships between the micro- and mesoquantities can be defined. In the future, this could provide an answer to the presently open question of how to model the coupling between transverse microcracking in the plies and damage in the interfaces.

\section{THE GENERAL HOMOGENIZATION PROBLEM}

\section{The reference model of the degraded laminate}

Let us consider the general case of a laminated plate. $N$ is the normal and $\pi$ is the projector onto the plane of the plate. The plate is subjected to a "macro" homogeneous loading defined by the plane part of the macrostrain $(\pi \varepsilon \pi)$ and the out-of-plane part of the macrostress $(\sigma \mathrm{N})$ (Figure 2).

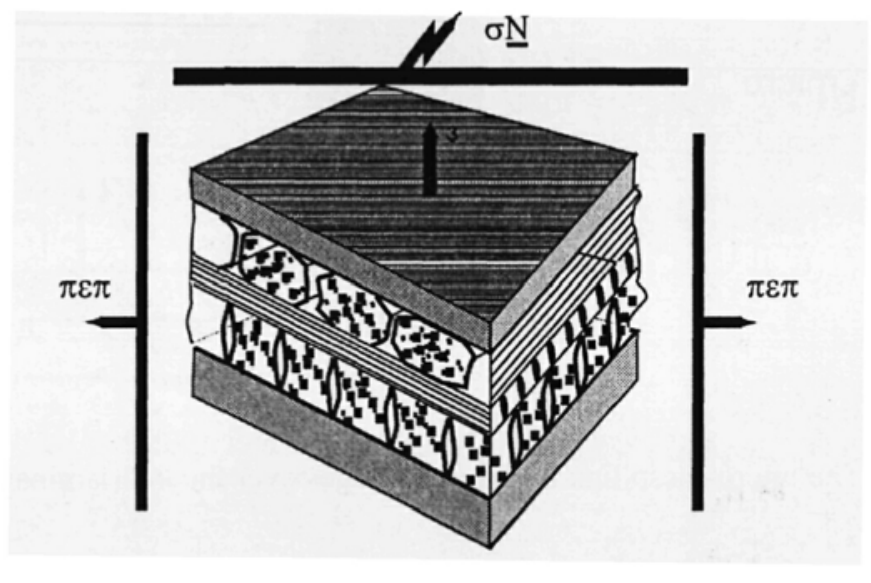

Fig. 2: The degraded laminate at the micro scale

The ply (i) is degraded by transverse cracking, measured by the microcracking density $\rho(i)$ (Scenario 1), and by diffuse delamination at the upper and lower interfaces $(\tau+(\mathrm{i}), \tau(\mathrm{i}))$ (Scenario 2$)$. [Alternatively, there can also be an initial level of diffuse debonding within the ply (Scenario 3).] The interface (j) is degraded on the microscale by diffuse delamination of the plies that it connects. 
With these hypotheses, one wishes to define the meso-equivalent laminate (in an energy sense), which is constituted only of homogeneous plies and interfaces.

Basis of the micro-meso link and associated mesoquantities

Let us call $\left(\sigma^{\text {meso }}, \varepsilon^{\text {meso }}\right)$ the mesosolution under the homogeneous macroloading defined above, and $\left(\sigma^{\text {micro }}, \varepsilon^{\text {micro }}\right)$ the microsolution. The latter is the superposition of two other solutions. Let us call the solution in the laminate without microcracks or diffuse delamination $(\bar{\sigma}, \bar{\varepsilon}) .(\bar{\sigma}, \bar{\varepsilon})$ is not the exact solution to the micro problem; residual stresses must be taken into account in the cracked areas. These residual problems define a correction $(\tilde{\sigma}, \tilde{\varepsilon})$ to the solution, such that:

$$
\left(\sigma^{\text {micro }}, \varepsilon^{\text {micro }}\right)=(\bar{\sigma}, \bar{\varepsilon})+(\tilde{\sigma}, \tilde{\varepsilon})
$$

One can prove that the mesoquantities can be interpreted as averages of the exact microquantities. In particular, for the ply (S) being studied, at the interface $\Gamma$ between the ply (S) and the adjacent ply, one can verify the properties defined in Figure 3:

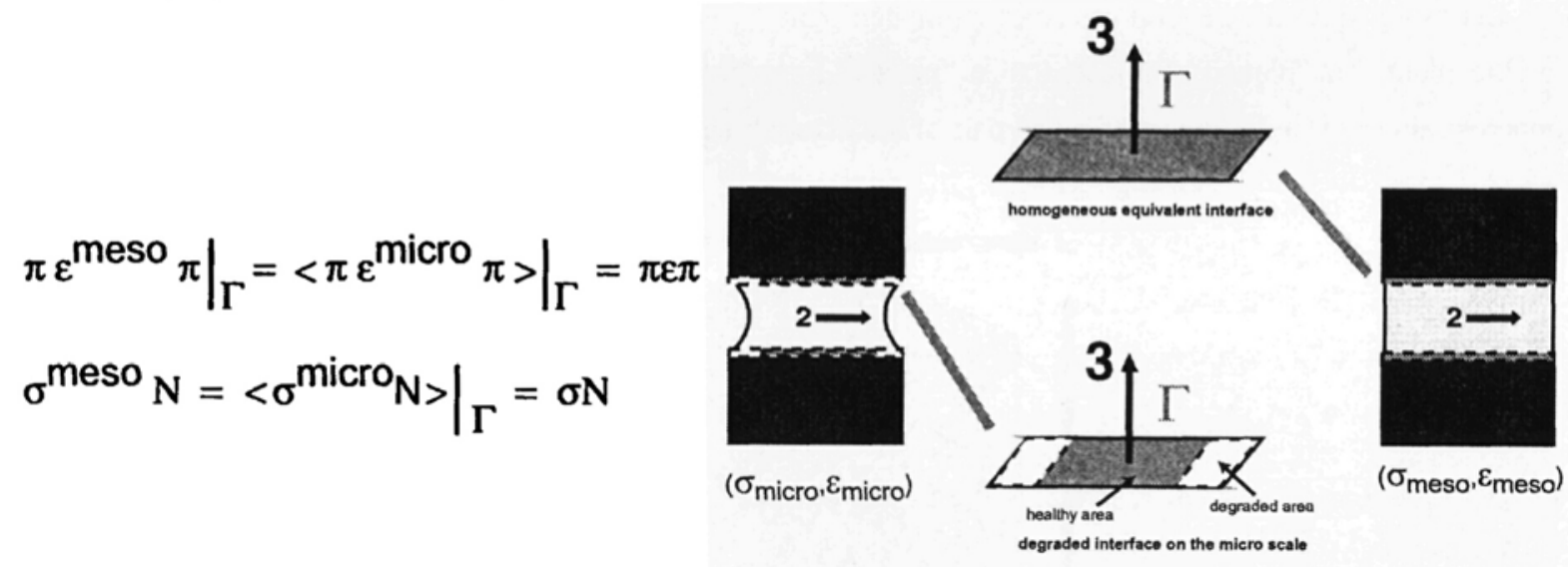

Fig. 3: The micro-meso link based on averages over the interlaminar interface

\section{DESCRIPTION OF THE TWO BASIC PROBLEMS}

\section{The approximate ply problem: $\mathbf{P}($ ply)}

In order to describe the induced problems easily, let us consider the case of a single ply affected by Scenarios 1 and 2 (Figure 4). 


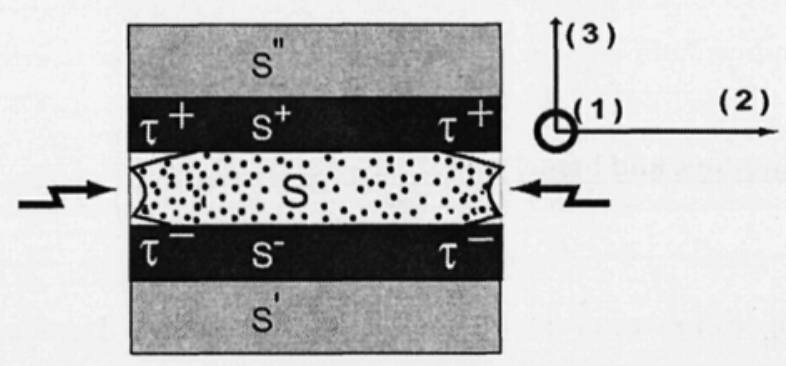

Fig. 4: The degraded ply on the microscale. The delaminated area is a fully damaged interface, whereas the central interface is healthy.

The ply can be homogenized. If the periodic cell is subjected to a homogeneous macro load case defined by $(\pi \varepsilon \pi, \sigma N)$, the potential energy in the degraded ply can be written as:

$$
\begin{aligned}
& 2 E_{p(S)} \stackrel{!}{=} \\
& \operatorname{mes}(S) \cdot\left\{[\pi \varepsilon \pi]^{t}\left[\tilde{M}_{1}\right][\pi \varepsilon \pi]+\sigma_{33}\left[\tilde{M}_{2}\right] \sigma_{33}+\sigma_{33}\left[\tilde{M}_{3}\right][\pi \varepsilon \pi]-\frac{\left(\bar{d}_{23}+1\right) \sigma_{23}^{2}}{\tilde{G}_{23}}-\frac{\left(\bar{d}_{13}+1\right) \sigma^{2}{ }_{13}}{\tilde{G}_{13}}-\frac{\bar{d}_{33}\left\langle\sigma_{33}\right\rangle^{2}}{\tilde{E}_{3}}\right\}
\end{aligned}
$$

where $M_{1}, M_{2}$ and $M_{3}$ are operators which depend on the in-plane damage indicators ([8], [9]) and on the properties of the initial homogeneous ply. (This initial ply can also be affected by homogeneous initial diffuse damage.) Consequently, the energy in the equivalent homogeneous ply is completely defined by five damage indicators (two in-plane, three out-of-plane). These indicators can be defined as integrals of the energy in the residual problem for the five basic residual load cases $\left(\sigma^{12} / \sigma^{22} / \sigma^{33} / \sigma^{13} / \sigma^{23}\right)$.

\section{The approximate interface problem: $\mathbf{P}$ (interface)}

Let us introduce for the interface the three residual problems of Figure 5. The loading on the interlaminar and intralaminar degraded areas is defined by $\sigma \mathrm{N}$.
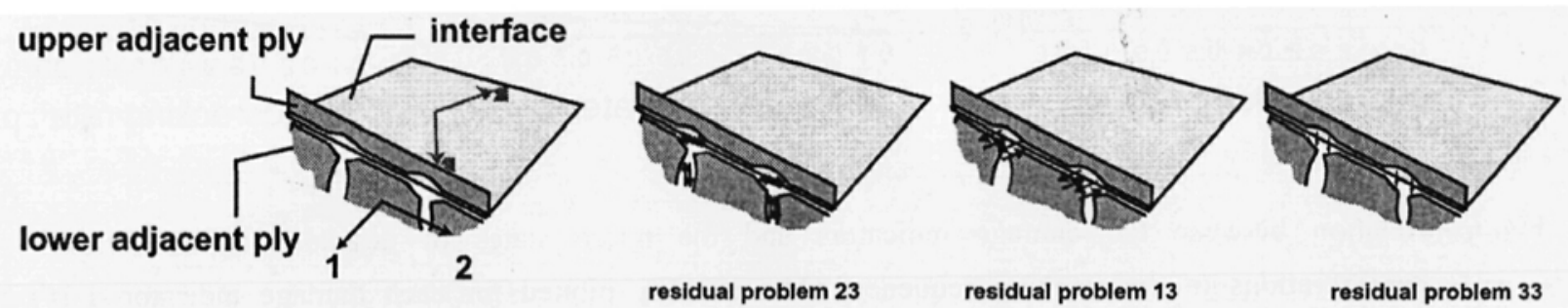

Fig. 5: The residual problems for the interface 
We can then assume that the strain energy of the interface under the macro homogeneous loading can be written as:

$$
2 E_{d}^{i}=2 E_{d 0}^{i}+\operatorname{mes}\left(\Gamma_{\mathrm{int}}\right) \cdot\left(\frac{\bar{d}_{33}^{i}}{\tilde{k}_{I}}\left\langle\sigma_{33}\right\rangle_{+}^{2}+\frac{\bar{d}_{13}^{i}}{\tilde{k}_{I I}} \sigma_{13}^{2}+\frac{\bar{d}_{23}^{i}}{\tilde{k}_{I I I}} \sigma^{2}{ }_{23}\right)
$$

where $\tilde{k}_{i}$ are the initial stiffnesses of the interface in the basis of the degraded ply, and $2 E_{d 0}^{i}$ is the energy in the healthy initial interface under the macro loading. Here again, the interface's damage indicators are linked to the strain energy of the micro interface in the residual problem.

Remark: here we are studying the influence of only one ply with diffuse delamination. If delamination occurs in both plies adjacent to the interface, the residual problem is actually a 3D problem with 2D periodicity.

\section{INTRINSIC PROPERTIES OF THE DAMAGE INDICATORS}

\section{Intrinsic relationships for the ply}

For various states of microdegradation (microcracking rates, diffuse delamination rates), equivalent damage indicators for the ply were calculated by finite-element resolution of the residual problems. For each degradation state, various configurations of the stacking sequence (orientations of the adjacent plies, relative thickness between plies, etc.) were studied.
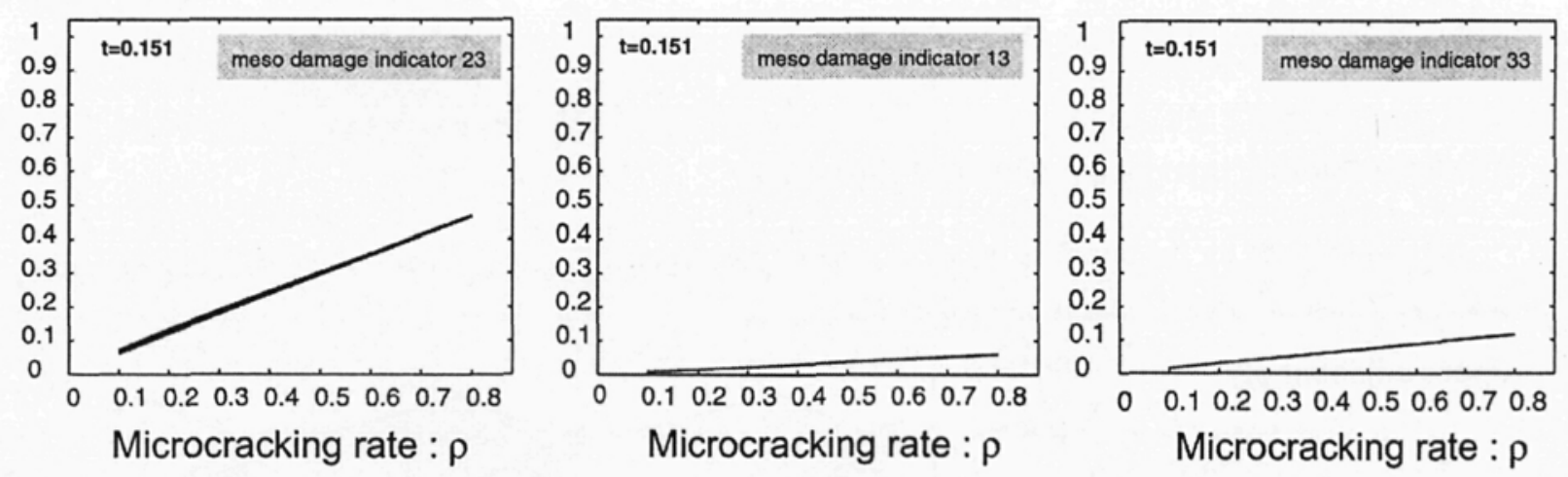

Fig. 6: Relation between the damage indicators and the micro states of degradation for various configurations of the stacking sequence. (The quantity plotted for each damage indicator $d$ is $d /(1+d)$.) 
Figure 6 shows values of the damage indicators versus the micro state for these different geometric configurations. It appears that an intrinsic link exists between the micro and meso measures of the degradation of the ply. This is in good agreement with the fundamental hypothesis of the mesomodel, which is that the characteristics of the mesoconstituents are intrinsic and do not depend on the stacking sequence.

\section{Intrinsic relationships for the interface}

Let us now consider an interface between two adjacent $0 \% 90^{\circ}$ plies (Figure 7). It is important to note that the damage indicators of the interface are approximately independent of the other, non-adjacent parts (S') and (S"). This property is the first consequence of the localization of the solution near the interface. However, it seems that other very important intrinsic properties exist. Work on this subject is in progress.

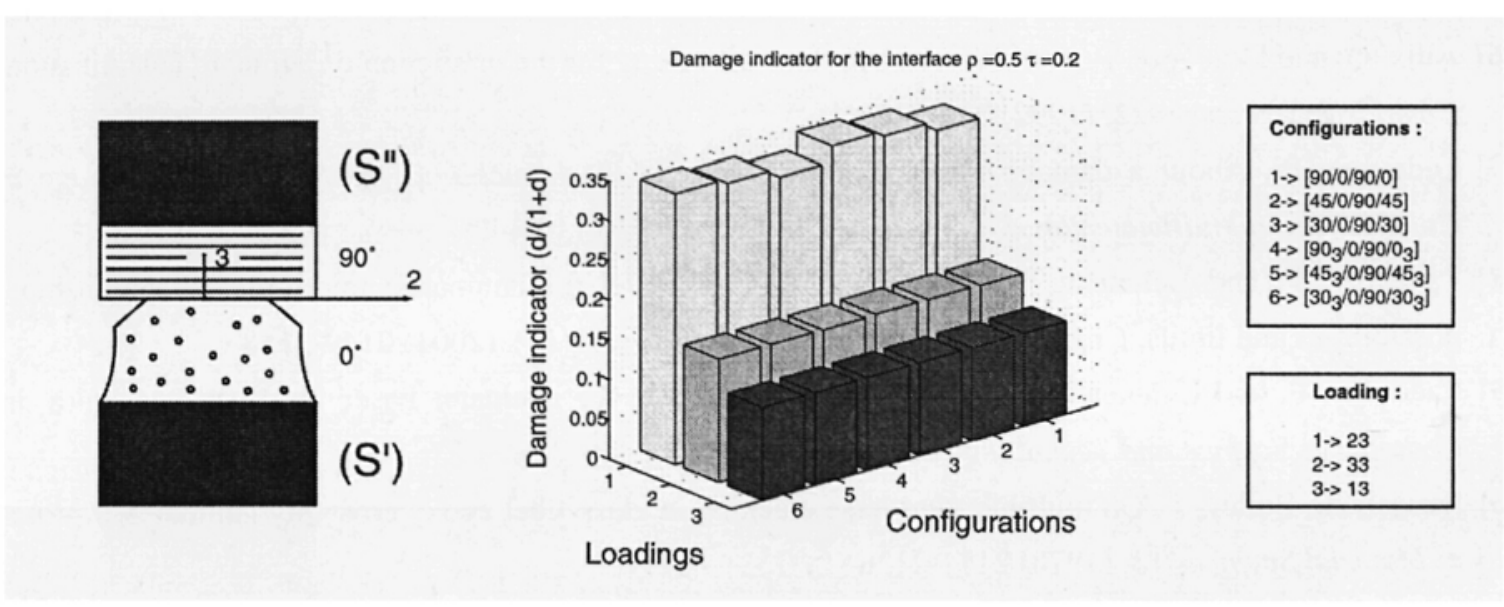

Fig. 7: dependence of the interface's damage indicators on non-adjacent plies.

\section{CONCLUSION}

We extended the micro-meso relations for laminates partially to the case of out-of-plane loading. Our numerical investigations show that there are intrinsic relations between the micro states of degradation and the damage indicators of the equivalent mesoconstituents. This seems to indicate again a good agreement between the mesoscale and the classical scenarios of degradation on the microscale. Nevertheless, further investigations are necessary to achieve a complete understanding of the micro-meso link under out-of-plane loading, especially with gradients. This work could eventually provide a solution to the problem of the modeling of the coupling between microcracking and delamination. 


\section{REFERENCES}

[1] Hashin, Z., Analysis of cracked laminates: a variational approach, Mechanics of Materials 4, (1985) $121-136$

[2] Nairn, J., Matrix microcracking in composites, in Polymer Matrix Composites, Taljera - Manson, eds., Ch. 13 (2000)

[3] Lagattu, F. and Lafarie-Frénot, M., Variation of PEEK matrix crystallinity in APC-2 composite subjected to large shearing deformation, Composites Science and Technology 60,(2000) 605-612

[4] Laws, N. and Dvorak, G.J., Progressive transverse cracking in composite laminates, Journal of Composite Materials 22, (1988) 900-916

[5] Ladevèze, P. and Le Dantec, E, Damage modelling of the elementary ply for laminated composites, Composites Science and Technology. 43(3), (1992) 257-267

[6] Allix, $O$. and Ladevèze, P., Interlaminar interface modelling for the prediction of laminate delamination, Composite Structures 22, (1992) 235-242

[7] Ladevèze, P., About a damage mechanics approach, in Mechanics and Mechanisms of Damage in Composite and Multimaterials, D. Baptiste, ed., MEP, (1989) 119-142

[8] Ladevèze, P. and Lubineau, G., On a damage mesomodel for laminates: micro-meso relationships, possibilities and limits, Composites Science and Technology 61(15), (2001) 2149-2158

[9] Ladevèze, P. and Lubineau, G., An enhanced mesomodel for laminates based on micromechanics, in Composites Science and Technology 62, (2002) 533-541

[10] Parvisi, A., Bailey, J., On multiple transverse cracking in glass-fiber epoxy cross-ply laminates, Journal of Material Sciences 13, (1978) 2131-2136

[11] Berthelot, J.M. and Le Corre J.F, A model for transverse cracking and delamination in cross-ply laminates, Composites Science and Technology, 60, (2000) 1055-1066 